BULL. AUSTRAL. MATH. SOC.

VOL. 34 (1986) 267-269

\title{
A NIL-IMPLIES-NILPOTENT RESULT IN ARTINIAN RINGS
}

\author{
J.H. Meyer
}

It is shown that if the ring $A$ is left Artinian and $L_{1}$ and $L_{2}$ are left ideals of $A$ then $L_{1}$ is nilpotent modulo $L_{2}$ if $L_{1}$ is nil modulo $L_{2}$.

An easy consequence of Levitzki's theorem is that if the ring $A$ is left Noetherian and $L$ is a left ideal which is nil modulo $I$, where $I$ is a two-sided ideal, then $L$ will be nilpotent modulo $I$. This can be proved by considering the ring $A / I$. The problem is: what if $I$ is a left ideal?

In this note I solve the problem for left Artinian rings, but a proof (or counter example) is still lacking in the left Noetherian case:

THEOREM Suppose the ring $A$ is left Artinian (not necessarily with 1). Let $L_{1}$ and $L_{2}$ be left ideals of $A$. Then $L_{1}$ is nil mod $L_{2}$ if and only if $L_{1}$ is nilpotent mod $L_{2}$.

Proof $\Leftarrow$ is trivial.

$\Rightarrow$ : Suppose $L_{1}$ is nil mod $L_{2}$. Consider the descending chain:

Received 22 November 1985.

Copyright Clearance Centre, Inc. Serial-fee code: 0004-9727/86 $\$ A 2.00+0.00$. 


$$
L_{1} \supseteq L_{1}^{2} \supseteq L_{1}^{3} \supseteq \cdots \geq L_{1}^{n}=L_{1}^{n+1}=B=B^{2}=\ldots
$$

Suppose $B^{2}=B \nsubseteq L_{2}$. Let $H$ be a minimal left ideal contained in $L_{1}$ such that $B H \notin L_{2}$. Then there is an element $h \in H$ such that $B h \notin L_{2}$. But now $B(B h)=B^{2} h=B h \nsubseteq L_{2}$ and $B h \subseteq H$ which forces $B h=H$ by the minimality of $H$. Let $b \in B$ be such that $h=b h$. Hence, $h=b h=$ $b^{2} h=\ldots=b^{q} h \in L_{2} h$ for $q$ large enough since $b \in B \subseteq L_{1}$ and $L_{1}$ is nil mod $L_{2}$. Consequently, there is an element $\ell \in L_{1} \cap L_{2}$ such that $h=l h$ and the following relation holds for all integers $i, j \geq 1$ :

$$
h^{j}=e^{i} h^{j}
$$

Let $p$ and $r$ be the smallest integers such that $(\ell+h)^{p} \in L_{2}$ and $h^{r} \in L_{2}(p, r \geq 2)$. Let $t=\max \{p, r\}$. Then

$$
\begin{aligned}
(l+h)^{t}=h & +k_{2} h^{2}+k_{3} h^{3}+\ldots+k_{r-1} h^{r-1}+k_{p^{2}} h^{r}+\ldots \\
& +h^{t}+\text { terms that end with an } l
\end{aligned}
$$

is an element of $L_{2}$, where the $k_{i}$ are integers. Hence

$$
h+k_{2} h^{2}+k_{3} h^{3}+\ldots+k_{r-1} h^{r-1} \in L_{2} \cdots
$$

If $r=2$, then ( $*$ ) would imply that $h \in L_{2}$, a contradiction. If $r>2$, then

$$
\begin{aligned}
& h^{r-2}\left(h+k_{2} h^{2}+\ldots+k_{r-1} h^{r-1}\right) \\
= & h^{r-1}+k_{2} h^{r}+\ldots+k_{r-1} h^{2 r-3} \in L_{2}
\end{aligned}
$$

which imply that $h^{r-1} \in L_{2}$, contradicting the minimality of $r$. Hence we must have $L_{1}^{n}=B \subseteq L_{2}$.

Examples suggest the validity of the result for left Noetherian rings ( with 1, of course). However, to the best of my knowledge this remains an unsolved open problem. 
[1] N. Divinsky, Rings and radicals, (University of Toronto Press, 1965).

[2] A.P.J. Van der Walt, "A variation on nil and nilpotent", Quaestiones Math. 7 (1984), 101 - 104.

Department of Mathematics

University of Stellenbosch

Stellenbosch 7600

South Africa. 